\title{
Caracterización espectroscópica de los sulfuros de zinc flotados en presencia de precipitados de hierro
}

\author{
J.A. Granados Oliver ${ }^{1 \star}$, M. Reyes Pérez¹, A.M. Teja Ruiz², M. Pérez Labra1 \\ F.R. Barrientos Hernández ${ }^{1}$, J.C. Juárez Tapia ${ }^{1}$, J.A. Cobos Murcia ${ }^{1}$
}

\begin{abstract}
${ }^{1}$ Área Académica de Ciencias de la Tierra y Materiales, Universidad Autónoma del Estado de Hidalgo, Mineral de la Reforma, Hidalgo, 42184, México. mreyes@uaeh.edu.mx, miguelabra@hotmail.com, frbh68@hotmail.com, jutj731101@hotmail.com, jose_cobos@uaeh.edu.mx

${ }^{2}$ Escuela Superior de Ingeniería Química e Industrias Extractivas ESIQUIE, Instituto Politécnico Nacional, Unidad profesional, Adolfo López Mateos. C.P. 07738, México, D.F. ice9791@gmail.com

*Autor de correspondencia: jago_madara@hotmail.com
\end{abstract}

\section{RESUMEN}

Durante la molienda de sulfuros minerales en molinos de bolas, los elementos molturadores se oxidan y forman una variedad de óxidos, hidróxidos y oxi hidróxidos de hierro; previas investigaciones han establecido que la adsorción de estas especies al mineral contamina la superficie y afectan la etapa de flotación. En esta investigación, se estudió la flotación de esfalerita en presencia de precipitados de hierro férrico y ferroso en diferentes concentraciones, los productos de la flotación se caracterizaron por espectroscopia de infrarrojo por transformada de Fourier. Los resultados muestran que el incremento de la flotación, depende de la formación de óxidos de hierro con una banda de enlace en $619 \mathrm{~cm}^{-1}$ correspondiente a goetita y de los óxidos de zinc con bandas de enlace del Zn-O en 1737, 1378, 1244, y 1031 cm-1, Se detectó además una banda en $1120 \mathrm{~cm}^{-1}$ indicando la formación de ion sulfato tetragonal libre. Similares resultados de flotación y composición superficial se obtienen cuando se tiene 75 ppm de ion férrico 0 ion ferroso. La esfalerita pulverizada en mortero de ágata, exhibió bandas de enlace del $\mathrm{Zn}-\mathrm{O}$, y la formación de sulfatos enlazados de manera mono dentada con el zinc con tres bandas de absorción.

Palabras Clave: Infrarrojo, Esfalerita, Ion Férrico, Ion Ferroso, Óxido de Zinc.

\section{INTRODUCCIÓN}

La flotación es un proceso fisicoquímico de separación, basado en diferencias superficiales entre los minerales de valor y la ganga que acompaña al mineral [1]. La mineralogía de los yacimientos minerales es compleja, generalmente, se encuentran en el yacimiento mineral varias especies como el sulfuro de cobre, sulfuro de plomo y el sulfuro de zinc, entre otros, los cuales deben separarse mediante flotación secuencial.

En las plantas concentradoras la flotación del sulfuro de zinc se lleva a cabo al final del proceso, por lo cual generalmente se emplean reactivos depresores tales como el sulfato de zinc, sulfito de sodio o cianuro de sodio [2], la flotación de esfalerita se efectúa empleando xantato y sulfato de cobre como activador a pH alcalino [3], no obstante, la flotación puede llevarse similarmente a pH ligeramente ácido [4]. Por otra parte, los iones plomo pueden activar la superficie de esfalerita y beneficiar la flotación $[5,6]$. 
El estudio del mecanismo de activación de esfalerita por los iones plomos se ha evaluado con la ayuda de espectroscopia fotoelectrónica de rayos X (XPS) y espectroscopia de infrarrojo por transformada de Fourier (FTIR). Concluyendo que la adición artificial de iones plomo en las pulpas de flotación logra incrementar la recuperación de esfalerita. De esta manera, se puede activar significativamente la esfalerita por los iones de plomo provenientes de la oxidación de sulfuros o especies minerales oxidadas de plomo [5].

Junto a esto, la activación superficial del mineral de esfalerita puede realizarse por los iones de metales presentes en el parámetro de red del mineral $[7,8]$. La esfalerita puede flotarse sin activación a pH menor a 6 donde los elementos contenidos en la superficie del mineral pueden solubilizarse para formar xantato de zinc $\left(Z n X_{2}\right)$. Otra investigación ha sugerido la activación con especies de hidróxido férrico en medio acido [9], proponiéndose mecanismos de activación bajo estas condiciones [10].

Se ha indicado previamente, que los iones $\mathrm{Fe}^{3+}$ derivados del mineral de esfaleritamarmatita oxidan al xantato para formar dixantato y lograr el éxito de la flotación [10]. Los trabajos anteriormente mencionados establecen el papel de la activación en la flotación de esfalerita, sin embargo, las investigaciones son escasas en el estudio de la espectroscopia de infrarrojo de los productos de flotación derivados de la activación del mineral con precipitados de $\mathrm{Fe}^{3+}$ y $\mathrm{Fe}^{2+}$.

En este trabajo de investigación se estudia la influencia del ion férrico $\mathrm{Fe}^{3+}$ y del ion ferroso $\mathrm{Fe}^{2+}$, provenientes de la sal de sulfato férrico y sulfato ferroso respectivamente en la activación y flotación sin colector de esfalerita usando únicamente espumante, considerando un amplio rango de concentraciones y su efecto en la eficiencia de la flotación y en el estado superficial del mineral, caracterizado mediante espectroscopia de infrarrojo por transformada de Fourier (FTIR).

\section{PARTE EXPERIMENTAL}

Para llevar a cabo este trabajo experimental, se usó un mineral de sulfuro de zinc, esfalerita ZnS puro, caracterizado previamente por técnicas instrumentales de DRX, MEB-EDS. La esfalerita se pulverizó en un mortero de ágata, se tamizó en seco usando una serie de tamices Tyler malla 170, 200, 270, 325, 400 y el fino. Las pruebas de flotación de esfalerita se llevaron en una celda de flotación tipo Denver usando para cada prueba $1 \mathrm{~L}$ de agua desionizada y partículas de mineral retenidas en la malla 200.

Para determinar el efecto de los precipitados de hierro $\mathrm{Fe}^{3+}$ y $\mathrm{Fe}^{2+}$ en la flotación de esfalerita, se emplearon, reactivos grado analítico tales como sulfato férrico $\mathrm{Fe}_{2}\left(\mathrm{SO}_{4}\right)_{3} \bullet \mathrm{H} 2 \mathrm{O}$ y sulfato ferroso $\mathrm{Fe}_{2} \mathrm{SO}_{4} \cdot 7 \mathrm{H}_{2} \mathrm{O}$ en concentraciones de $5,25,50$ y 75 ppm individualmente, para la disminución de la tensión superficial de la pulpa se empleó espumante, del tipo aceite de pino en concentración de 60 ppm proveído por la empresa Alkemin S.A de S.R.L. Se emplearon tiempos de flotación de 30 segundos, 1, 2, 4, 6, 8 y 10 minutos obteniendo las curvas recuperación versus el tiempo de flotación.

Durante la etapa de acondicionamiento de la pulpa y al final de la flotación se monitoreo el potencial de iones hidrogeno $(\mathrm{pH})$, el potencial óxido-reducción (ORP) y la conductividad eléctrica $(k)$. Los concentrados obtenidos a diferentes tiempos de flotación 
30 segundos y 1 minuto se caracterizaron por FTIR para determinar el estado superficial de las partículas minerales durante la flotación sin colector de esfalerita.

\section{RESULTADOS}

La Figura 1 muestra las micrografías de MEB de las partículas de esfalerita en general y a detalle, se observan partículas de morfología irregular, de bordes alargados y de apariencia en capas con cantos lisos. Del microanálisis por dispersión de energías (EDS) el mineral presenta la siguiente composición química semi cuantitativa: $54.46 \%$ Zn, 26.26 \% S, 14.59 \% Fe y 4.69 \% de oxígeno. La Figura 2 muestra el análisis de difracción de rayos $X$ del mineral empleado en esta investigación se observa que se trata de una sola fase cristalina de sulfuro de zinc identificado con el patrón de difracción PDF 00-0050566.
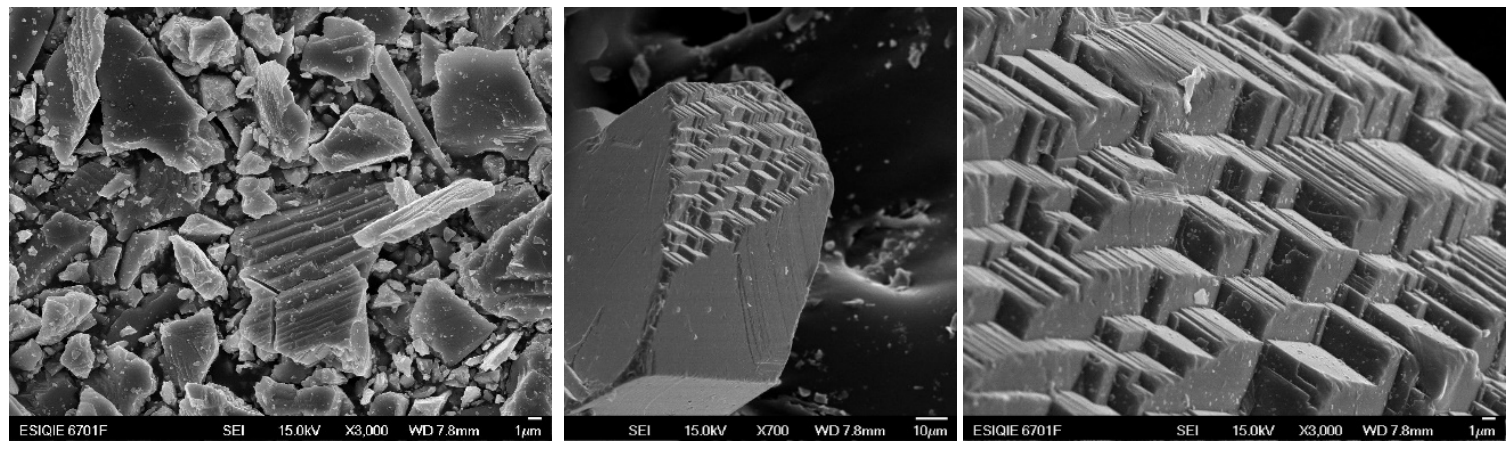

Figura 1. micrografías de $M E B$, imagen general y a detalle de las partículas de esfalerita.

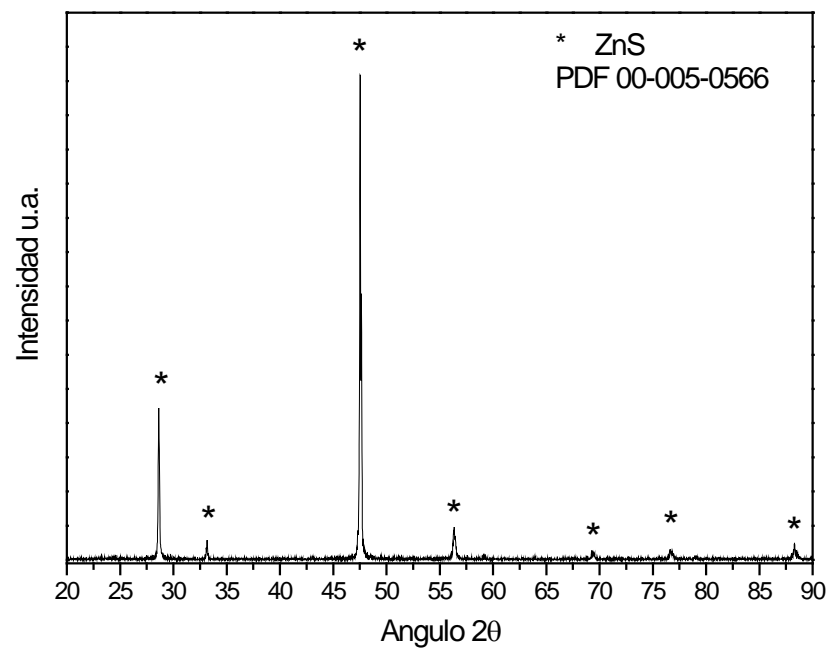

Figura 2. Espectro de difracción de rayos $X$ del mineral de esfalerita.

Los resultados de las pruebas de flotación sin colector, del mineral de esfalerita a pH 6.36, empleando $60 \mathrm{ppm}(\mathrm{mg} / \mathrm{L})$ de espumante aceite de pino, y concentraciones de 5, 25, 50 y 75 ppm de ion férrico $\mathrm{Fe}^{3+}$, se muestran en la Figura 3, generalmente la recuperación acumulativa del mineral incrementa con el tiempo de flotación obteniendo alrededor del 99 \% de separación de esfalerita en un tiempo de 10 minutos, y con una concentración de ion férrico de 75 ppm. 
El hierro $\mathrm{Fe}^{3+}$ en la pulpa a $\mathrm{pH}$ de 6.3 precipita como hidróxido férrico $\mathrm{FeO} \cdot \mathrm{OH}$ (goetita) tal como lo establece la termodinámica, la Figura 4 muestra el diagrama Eh-pH del sistema Fe- $\mathrm{H}_{2} \mathrm{O}$ a $25{ }^{\circ} \mathrm{C}$, obtenido del software HSC Chemistry V5.11. Donde el hierro en las condiciones de flotación precipita como hidróxido férrico (goetita).

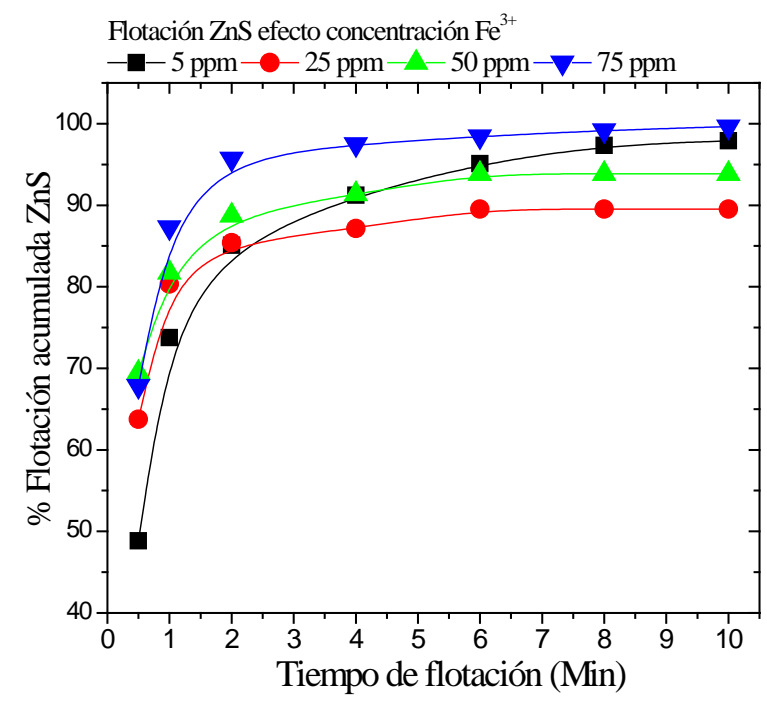

Figura 3. \% de flotación acumulada de ZnS vs tiempo de flotación para concentraciones de ion férrico de 5, 25, 50 y 75 ppm

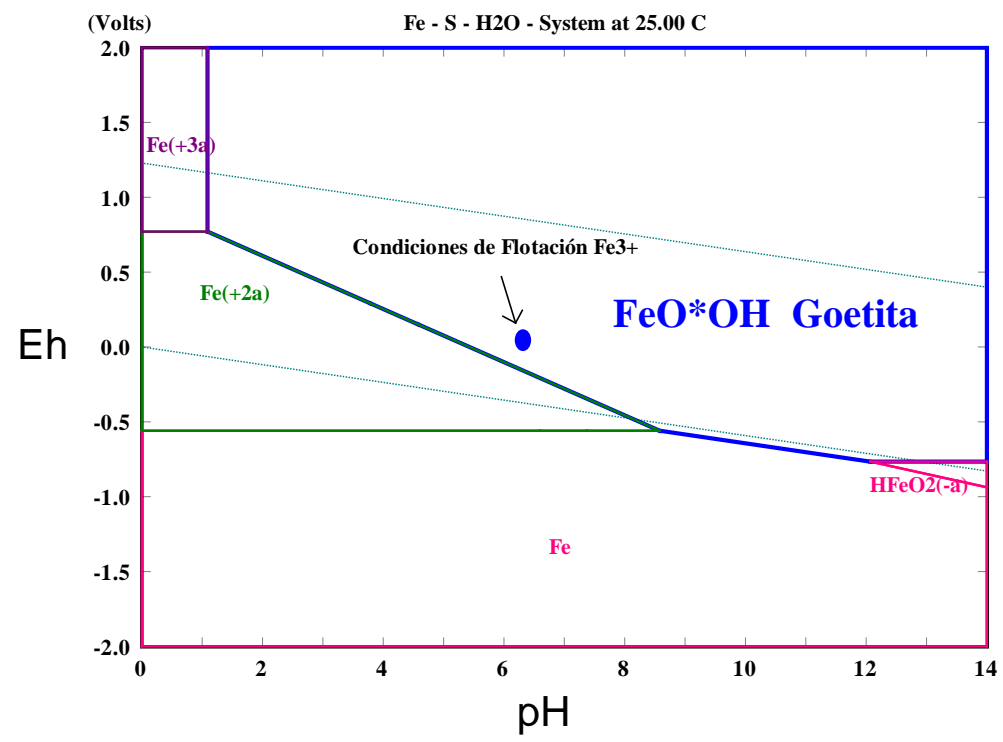

Figura 4. Diagrama de Eh-pH para el sistema $\mathrm{Fe}-\mathrm{H}_{2} \mathrm{O}$ para $1.3 \times 10^{-3} \mathrm{~m}$ de $\mathrm{Fe}^{3+}$

El análisis por espectroscopia de infrarrojo de los sólidos de esfalerita flotados en los primeros 30 segundos y 1 minuto, para las pruebas con $75 \mathrm{ppm}$ de ion férrico $\mathrm{Fe}^{3+}$ se muestran en la Figura 5. Esta figura contiene además el espectro de infrarrojo de los enlaces $\mathrm{Zn}-\mathrm{O}$ de un reactivo químico puro de óxido de zinc. La banda de absorción en alrededor de $619 \mathrm{~cm}^{-1}$ corresponde a los enlaces Fe-O de la especie química precipitada de hierro goetita [10], cabe mencionar que esta banda de enlace no se detectó en los espectros de FTIR de la esfalerita flotada empleando como activador superficial ion férrico en concentraciones de 5, 25 y 50 ppm. Por otra parte las bandas de enlace en 
$1031 \mathrm{~cm}^{-1}, 1244 \mathrm{~cm}^{-1}, 1378 \mathrm{~cm}^{-1}$ y $1737 \mathrm{~cm}^{-1}$, especialmente para la esfalerita flotada en los primeros 30 segundos corresponden a los enlaces $\mathrm{Zn}-\mathrm{O}$.

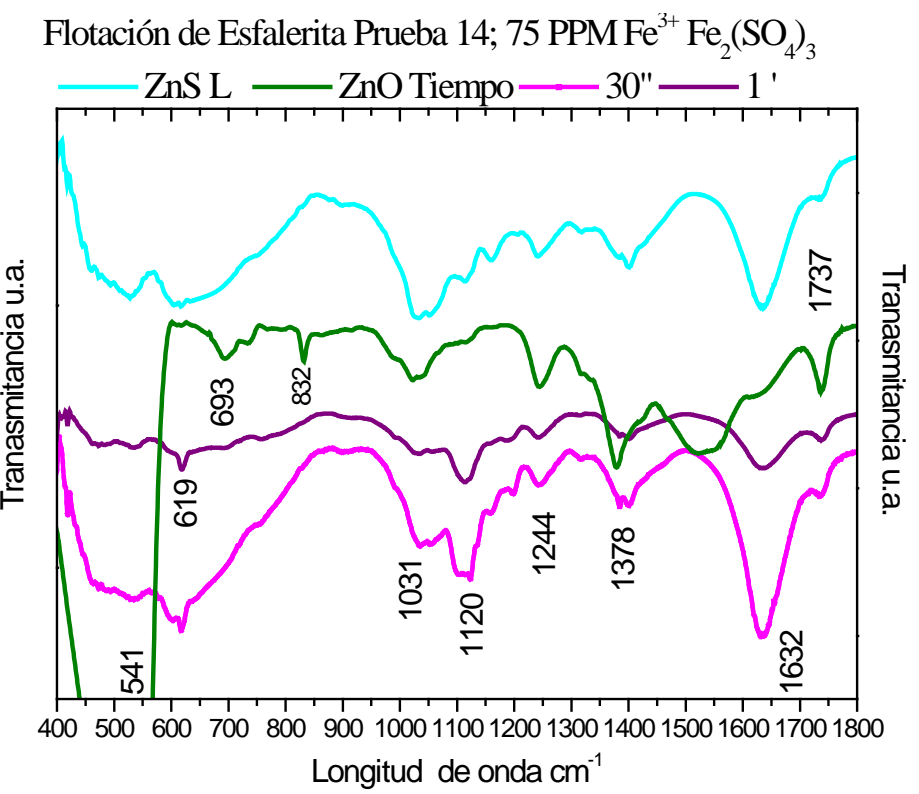

Figura 5. Espectros de infrarrojo por transformada de Fourier de la esfalerita flotada usando 75 ppm de ion férrico, a los 30 segundos y 1 minuto, y de los enlaces $Z n-O$ del óxido de zinc.

Por lo tanto, la presencia de los enlaces $\mathrm{Zn}-\mathrm{O}$ y Fe-O en la superficie de la esfalerita son esenciales para el éxito de la flotación sin colector. En $1120 \mathrm{~cm}^{-1}$ se tiene una banda de absorción correspondiente al ion sulfato tetragonal libre es decir no se forman enlaces con el metal en la superficie del mineral.

La Figura 6 muestra el \% de flotación acumulativa vs tiempo, empleando concentraciones de 5, 25, 50, y 75 ppm de ion $\mathrm{Fe}^{2+}$, empleando sal de sulfato ferroso. Tal como en el caso anterior, las mejores eficiencias se flotación se logran cuando se tienen 75 ppm de $\mathrm{Fe}^{2+}$, con alrededor del 99 \% y prácticamente en los dos primeros minutos de flotación se logra separar la mayor cantidad de esfalerita.

Los sólidos obtenidos durante la flotación de esfalerita empleando ion ferroso se caracterizaron por FTIR. La Figura 7 muestra los espectros de infrarrojo para la esfalerita flotada en presencia de precipitados de hierro provenientes de la sal de sulfato ferroso $75 \mathrm{ppm}$ de hierro ferroso. Las bandas de enlace en $1026 \mathrm{~cm}^{-1}, 1244 \mathrm{~cm}^{-1}, 1378 \mathrm{~cm}^{-1} \mathrm{y}$ $1737 \mathrm{~cm}^{-1}$, indican la presencia de los enlaces $\mathrm{Zn}-\mathrm{O}$ del óxido de zinc formado sobre la superficie del mineral, así como el enlace $\mathrm{Fe}-\mathrm{O}$ del $\mathrm{FeO} \cdot \mathrm{OH}$ correspondiente a la goetita, con la banda de enlace en $619 \mathrm{~cm}^{-1}$. 


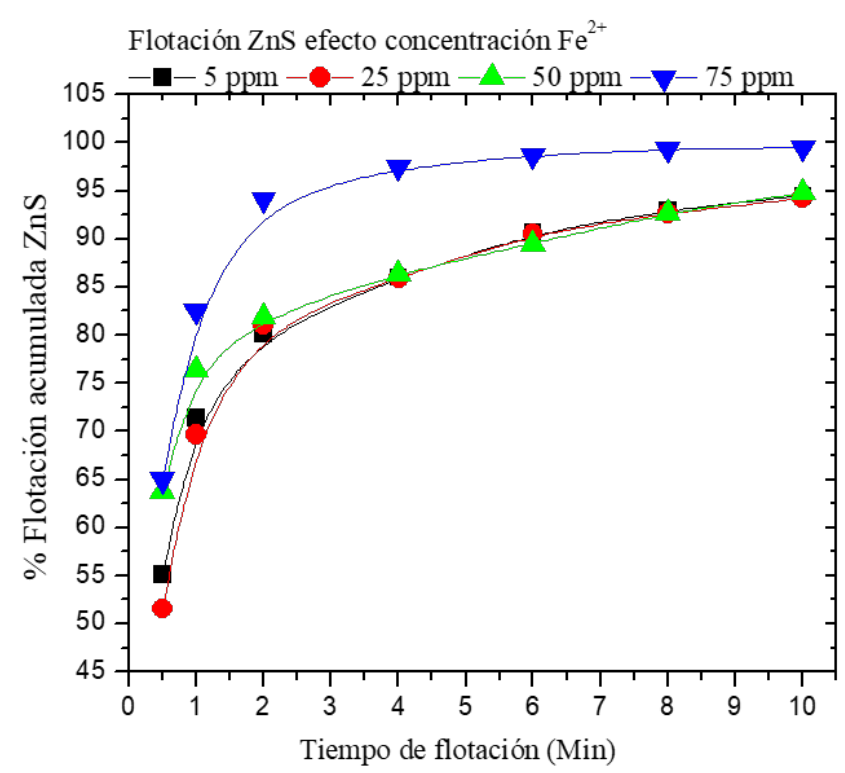

Figura $6 \%$ de flotación acumulada de ZnS vs tiempo de flotación para concentraciones de ion ferroso $\mathrm{Fe}^{2+}$, con 5, 25, 50 y 75 ppm usando una sal de sulfato ferroso.

Flotación de Esfalerita Prueba 18; $75 \mathrm{PPM} \mathrm{Fe}^{2+} \mathrm{FeSO}_{4} * 7 \mathrm{H}_{2} \mathrm{O}$

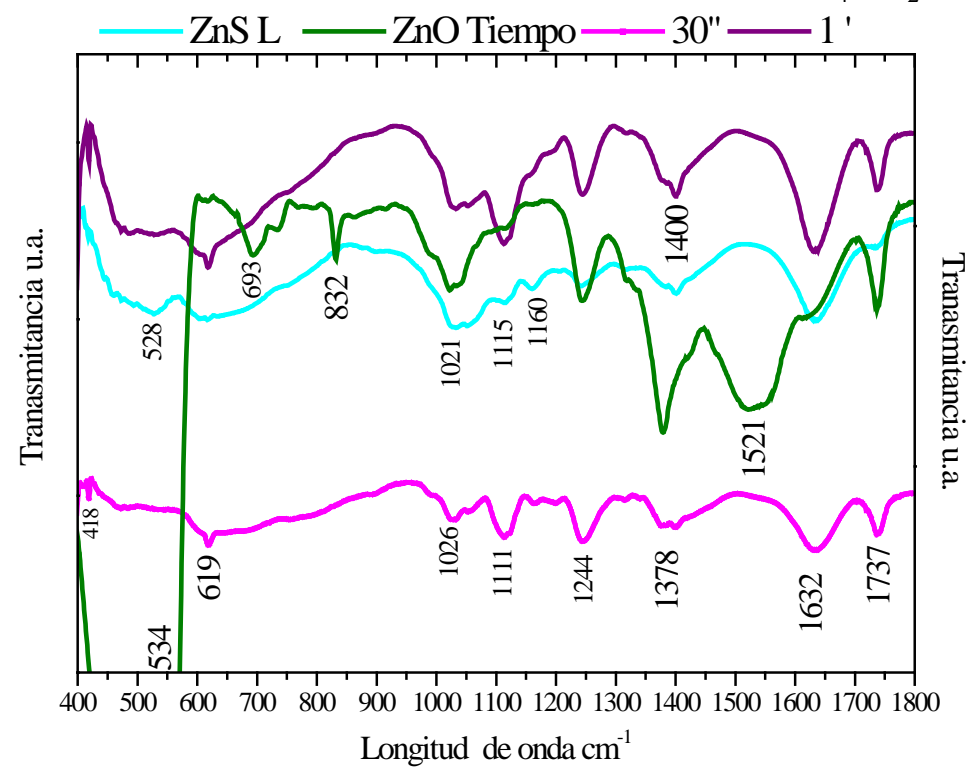

Figura 7. Espectros de infrarrojo por transformada de Fourier de la esfalerita flotada usando 75 ppm de ion ferroso, 30 segundos y 1 minuto.

\section{CONCLUSIONES}

El mineral empleado en esta investigación es uno de alta pureza con contenidos de hierro significativos los cuales de alojan en el parámetro de red. La flotación de esfalerita se puede efectuar exitosamente sin el uso de colector y con $60 \mathrm{ppm}$ de espumante usando como reactivos activadores de superficie tanto ion férrico como ion ferroso individuamente, proveniente de sus sales de sulfato. Las fuertes bandas de enlace del 
Zn-O y Fe-O detectadas en los espectros de infrarrojo cuando la flotación se efectúa con 75 ppm de ya sea $\mathrm{Fe}^{3+}$ o $\mathrm{Fe}^{2+}$ mejoran significativamente la recuperación acumulativa de esfalerita.

\section{AGRADECIMIENTOS}

A la Universidad Autónoma del Estado de Hidalgo, al Programa para el Desarrollo Profesional Docente PRODEP, y al Instituto Politécnico Nacional unidad ESIQUIE.

\section{BIBLIOGRAFÍA}

[1] B.A. Wills, J.A. Finch, Introduction, in: B. A. Wills, J. A. Finch (Eds.), Wills' Miner. Process. Technol., Eighth Ed, Elsevier, Boston 2016, pp. 1-27.

[2] S. Song, A. Lopez Valdivieso, J. L. Reyes Bahena, C. Lara Valenzuela. Miner. Eng. 14 (2001) 87-98.

[3] W.Z. Shen, D. Fornasiero, J. Ralston. Miner. Eng. 11 (1998) 145-158.

[4] S.L. Harmer, A. Mierczynska-Vasilev, D.A. Beattie, J.G. Shapter. Miner. Eng. (2008) 21, 1005-1012.

[5] C.I. Basilio, I.J. Kartio, R.H. Yoon. Min. Eng. 9 (1996) 869-879.

[6] R. Houot, P. Ravenau. Int. J. Miner. Process. 35 (1992) 253.

[7] N.P. Finkelstein, S.A. Allison. M.C. Fuerstenau. Flotation, A.M. Gaudin Memorial Volume (1976) SME/AIME, New York, 414.

[8] B. Marouf, J. Bessiere, R. Houot, P. Blazy. Trans. Inst. Min. Metall. (1986) 95 83-88.

[9] M. Leroux, S.R. Rao, J.A. Finch. CIM Bull. 80 (1987) 41-44.

[10] K. Nakamoto. Infrared and Raman Spectra of Inorganic and Coordination Compounds. 3rd ed. 1978: Wiley, New York. 